\title{
Analysis on the Characteristics of Ordinary and More Serious Accidents in Building Projects in China from 2005 to 2016
}

\author{
Hui Zhang , Xuewei Ji and Aizhi Wu \\ Beijing Academy of Safety Science and technology, Beijing 100070, China
}

\begin{abstract}
In China, the frequent safety accidents in building projects seriously affect the sustainable development of construction projects. The paper makes a statistical analysis on the characteristics of ordinary and more serious accidents in building projects from the types of accidents, the trends of development and the characteristics of geographical distribution from 2005 to 2016 in China by using the histogram, trend chart, pie chart and table. It can be concluded that in the recent 12 years, the security situation of construction projects generally fluctuates in a big way and is still not optimistic. Among the five major types of accidents, the electric shock and the lifting accidents have been maintained at a relatively stable and low incidence, and the sloughing accidents have accounted for more than half of the proportion. Accidents concentrated in East China, Central China, North China and Southwest China. The economic development in all provinces and cities in China is positively related to the accident. The greater the total regional economy, the rate of economic growth and the demand for infrastructure construction, the more deaths and accidents in construction projects in the corresponding regions.
\end{abstract}

\section{Introduction}

The large-scale construction projects in China have employed a large number of workers which are the largest working groups in the world [1]. Since 2001, macro-economy has entered a new round of ascent cycles, and the number of construction projects has also skyrocketed in China. In the Construction Engineering, which is a branch of China's construction industry, the data published by the National Bureau of Statistics shows that, the added value of China's construction industry amounted to 17.8591 trillion yuan from 2003 to 2012 [2], the number of employees increased from 38.5247 million in 2011 to 43.5679 million in 2015 , and the total output value increased from 11.663332 trillion yuan in 2011 to 18. 757 trillion yuan in 2015 [3]. However, in fact, China's construction projects also include civil engineering, pipeline and equipment installation and renovation works. With the expanding scope and scale of engineering construction in our country, the natural environment, construction types and structures confronted by construction are more diversified, increasing the engineering construction technology challenge [4], as a result that the number of accidents and casualties have not been effectively contained, causing great economic losses to the construction and negatively affecting the social harmony and stability. Therefore, combining the data of construction safety accidents occurred in China in recent years, this paper analyzes the type characteristics, geographical features and development trends of the accidents, which have reference and guidance significance to the control and prevention of construction accidents in China.

Relevant experts and scholars in the statistical analysis of construction safety accidents in China have made a series of achievements: Li Yanjie, Yang Liqiong and others [5] count the accidents occurred in China's construction industry from 2007 to the first half of 2017, analyze the regulation of the accident from the accident type, the occurrence time and the occurrence season, basing on the overall status of the accidents which have occurred, and reached a conclusion. Wu Yongfeng [1] analyzes the security incidents occurred in China from 2004 to 2012, through charts, histograms, pie charts and other forms, finds the law of accident and puts forward specific suggestions on the safety management of the industry. Zhang Tong and others [6] analyze building accidents in China from 2003 to 2012, from the correlation between the characteristics of the accident and economic development trends, and put forward corresponding improvement measures for the prevention and control of the construction accidents. Hao Zhong and Wei Yanxiao [7] analyzed the rules and characteristics of the 448 accidents from the degree of injury, type of injury, working type of accident and so on, and put forward measures and suggestions to prevent and control occupational injuries, basing on the rules and characteristics of the accident. Yuan Shuang [8] collectes 8293 safety accidents occurred in China's municipal housing project from 2003 to 2012., with "The People's Republic of China Ministry of Housing and Urban-Rural 
Construction Injury Express Letters" as the core data source, extracts the time, region, grade, etc. from the accident to establish an absolute index system, at the same time, obtains regional construction output and practitioners'number from the China Statistical Yearbook and local statistical yearbook in 2003-2012, and calculats the output index of 10 billion yuans and the death rate of 100,000 employees, establishing a relative index system to conduct a comprehensive evaluation on the fluctuation characteristics and development trend of the management level of safety accident. Wang Dan, Cui Li Miao and others analyze the construction accidents information in China from 2011 to 2015 by using SPSS in terms of the month, time period, provinces and types of accidents, and propose measures to reduce the construction accidents in China to provide a reference for safety management of building enterprise. Based on the theory of safety system engineering, safety management engineering and safety evaluation, Sun Jian [9] analyzes the characteristics of safety accidents that may occur during the construction operations and discusses the safety management and comprehensive evaluation of construction projects from the perspective of man-machine-environment, using analytic hierarchy process and gray comprehensive evaluation method. Shui Zhengxiang [10] automatically collectes and manages 318 typical cases of major accidents in China from 2005 to 2014 and extractes accident keywords by using software and analyzes the distribution characteristics from time, accident type and region by using statistical analysis method to find the law of the accident occurrence, with "The notification of production safety accident in housing municipal engineering " issued by Ministry of Housing and Urban-Rural Development of the People's Republic of China as the main source of data.

Based on the data of construction accidents obtained from the Accident Inquiry System of the State Administration of Work Safety, this article counts 520 ordinary and more serious construction accidents in China from 2005 to 2016, including the construction of bridges, railways and ports, housing construction projects and underground tunnel construction, and explores the space-time characteristics and development trend of the accidents from time, type and geographical area to guide the construction and prevention of ordinary and more serious construction accidents in China.

\section{The basic characteristics of ordinary and more serious construction accidents}

According to the types of accidents including falling, collapse, object strike, lifting injury, electric shock and others, this section calculated the number of accidents and deaths from ordinary and more serious construction accidents in China during 2005-2016 to get the trend of the total number of accidents deaths. The statistical results are shown in Table 1.

It can be seen from Table 1 and Figure 1 and Figure 2 that the number of accidents and deaths of ordinary and more serious construction accidents in China from 2005 to 2016 have not shown the increasing or decreasing trend regularly and have fluctuated obviously. In recent 12 years, the trend of construction engineering in China has the following characteristics:

Table 1. The statistics table of ordinary and more serious construction accidents in China from 2005 to 2016.

\begin{tabular}{ccccc}
\hline Year & $\begin{array}{c}\text { The } \\
\text { number of } \\
\text { accidents }\end{array}$ & $\begin{array}{c}\text { Accident } \\
\text { reduction } \\
\text { rate } \%)\end{array}$ & $\begin{array}{c}\text { The } \\
\text { number } \\
\text { of deaths }\end{array}$ & $\begin{array}{c}\text { The } \\
\text { decreasi } \\
\text { ng rate } \\
\text { of } \\
\text { deaths } \\
(\%)\end{array}$ \\
\hline 2005 & 40 & - & 152 & - \\
2006 & 46 & -15.0 & 168 & -10.5 \\
2007 & 36 & 21.7 & 148 & 11.9 \\
2008 & 52 & -44.4 & 214 & -44.6 \\
2009 & 40 & 23.1 & 169 & 21.0 \\
2010 & 55 & -37.5 & 245 & -45.0 \\
2011 & 47 & 14.5 & 187 & 23.7 \\
2012 & 42 & 10.6 & 184 & 1.6 \\
2013 & 44 & -4.8 & 170 & 7.6 \\
2014 & 51 & -15.9 & 194 & -14.1 \\
2015 & 31 & 39.2 & 118 & 39.2 \\
2016 & 36 & -16.1 & 222 & -88.1 \\
Total & 520 & - & 2171 & - \\
\hline
\end{tabular}

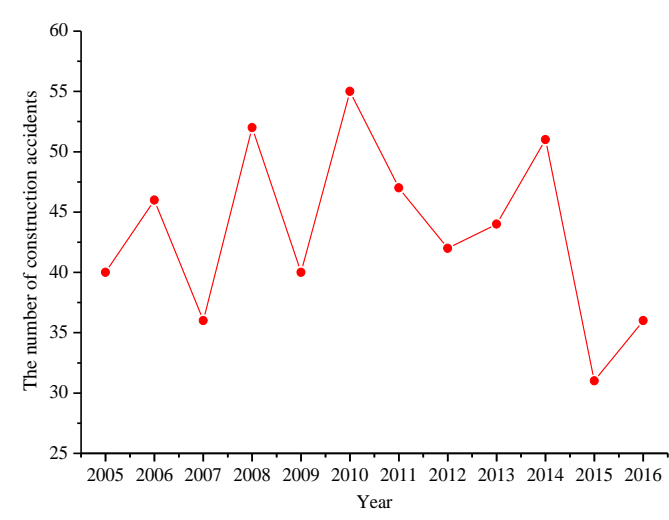

Figure 1. The trend of the number of accident.

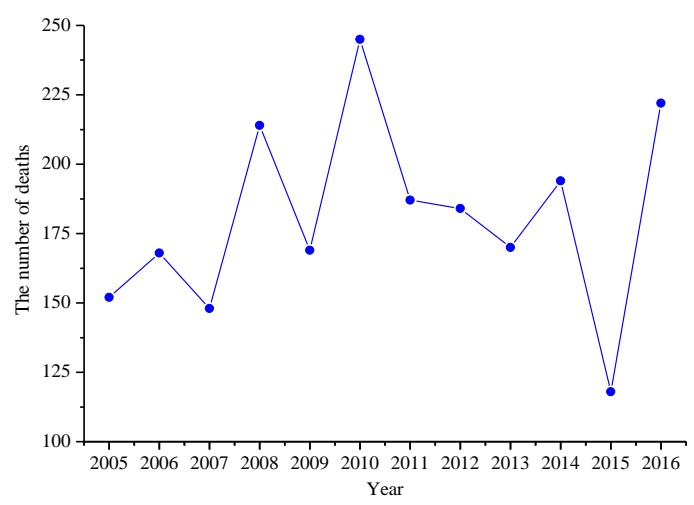

Figure 2. The trend of the number of deaths. 
(1) In the ordinary and more serious construction accidents in China from 2005 to 2016, the trend of the total number of accidents and deaths is almost the same. In other words, the total number of deaths in the year also increased when the total number of ordinary and more serious construction accidents increased in a year was larger than that in the previous year; When the total number of accidents in a year decreased, the total number of deaths corresponding to the year also decreased compared with that of the previous year. However, with the exception of the statistics in 2013, the number of accidents increased during the year but that of deaths decreased compared to 2012.

(2) The total number of accidents and deaths in 2010 was the highest in 12 years between 2005 and 2016, with a $37.5 \%$ increase in accidents and a $45 \%$ increase in deaths compared to 2009, showing that in 2010, the construction safety inChina is extremely poor. Compared with the data in 2010, accidents from 2005 to 2010 can be assumed to show a increasing trend year by year.

(3) The incidence of accidents between 2010 and 2016 can be approximated as showing a downward trend. In particular, a good construction safety was shown in 2015, with a drop of $39.2 \%$ in the number of accidents and deaths in the year, which is the lowest annual accident count and death tolls in the past 12 years. Although showing a slight rebound in 2016 compared with 2015 , these two indicators generally remained at a relatively low level.

The number of construction accidents has fluctuated rather than showing a steady decline since 2005. But the number of accidents and deaths in 2015 and 2016 have dropped significantly compared with the previous 10 years, which showed the results of strengthening construction safety management in China in recent years. However, the occurrence of accidents has not been fundamentally deterred, which is the key that restricts the increasing of the safety management and construction in China.

\section{Type characteristics of the accident}

"Lifting injury, falling, objects strike, electric shock, collapse" is known as the five major construction safety injuries. In the ordinary and more serious construction accidents in China from 2005 to 2016, in addition to object strike, the four major types of injuries occupied the major part of the accident. In order to analyze the annual variation trend of different accident types and the changes of death toll better, this section classified and counted the ordinary and more serious construction accidents in China in the past 12 years. The chart of accident types the annual trend of the different types of accidents are shown in Figure 3 to 6.

According to the statistical results, only considering collapse, object strike, lifting injury, electric shock and falling, 307 ordinary and more serious construction accidents have occurred, with 1,322 deaths in the past 12 years. Among these, there were 197 collapse accidents and 858 deaths in them, accounting for $64 \%$ and $65 \%$ of the total respectively. There were 84 falling accidents and
372 people died in them, accounting for $27 \%$ and $28 \%$ respectively. There were 15 lifting injuries and 56 people died, accounting for $5 \%$ and $4 \%$ respectively. There were 11 electric shock accidents and 36 deaths, accounting for $4 \%$ and $3 \%$ respectively.

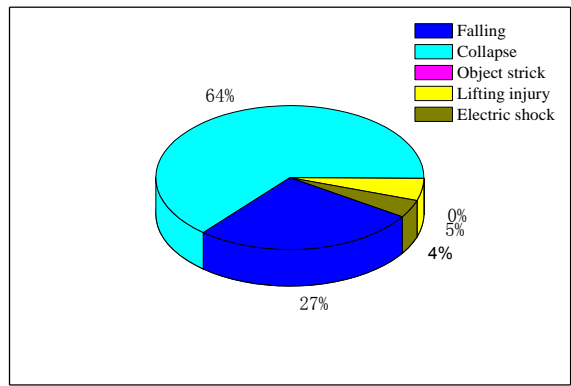

Figure 3. Scale of accident type.

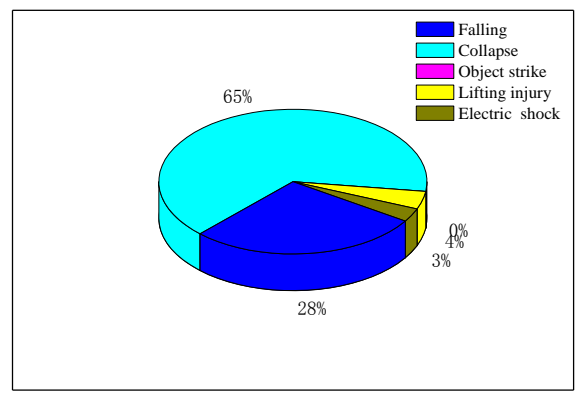

Figure 4. Annual trend of the different accidental types.

It can be seen from Figure 3 and Figure 4 that the collapse accidents all took the first place, far exceeding those of the other three Kinds of accidents, no matter the number of accidents or the number of deaths, among the five major types of construction accidents during 2005-2016. Fallings, lifting injuries and electric shock accidents came in second, third and fourth respectively. Thus, collapse accident is the most common form of injury and the easiest event to happen in construction, but also the focus of accident prevention.

As can be seen from Figure 5 and Figure 6, the number of deaths, involving falling accidents, lifting injuries and electric shock accidents, showed the same trend with the number of accidents in each year. Collapse accidents were the most volatile, especially in 2015 and 2016. There were 22 collapse accidents in 2015 with 88 deaths and 16 collapse accidents with 136 deaths in 2016. It can be seen that although the number of accidents increased slightly in 2015 as compared with 2014, the total number of deaths decreased, indicating that the seriousness of accidents in 2015 was not high. And although the number of accidents in 2016 markedly decreased, the total number of deaths increased significantly, indicating that most of the accidents occurred in 2016 were destroyous accident and tremendous devastating accident, indicating thatthe construction safety situation in China remains very serious. From the trend chart, it can be seen that, for electric shocks and lifting accidents from 2005 to 2016, the fluctuations in the number of accidents and the deaths 
are both very small and at a relatively low and stable level. The annual average number of accidents and deaths in lifting accidents are 2 and 5, and those in electric shocks are 1 and 3. Due to the fact that collapse accidents occupy a larger proportion in all types of accidents than before, the focus of construction safety management should be on the prevention of collapse accidents and falling accidents in the future.

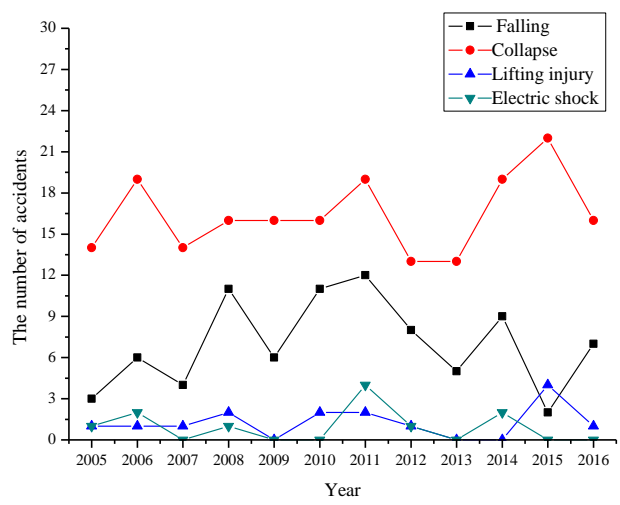

Figure 5. Annual trend of various accidental types.

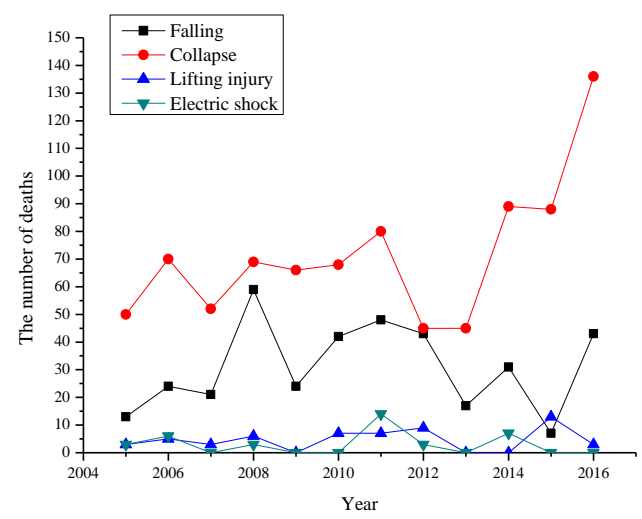

Figure 6. Trend of death toll of each accident type.

\section{Regional characteristics of the accident distribution}

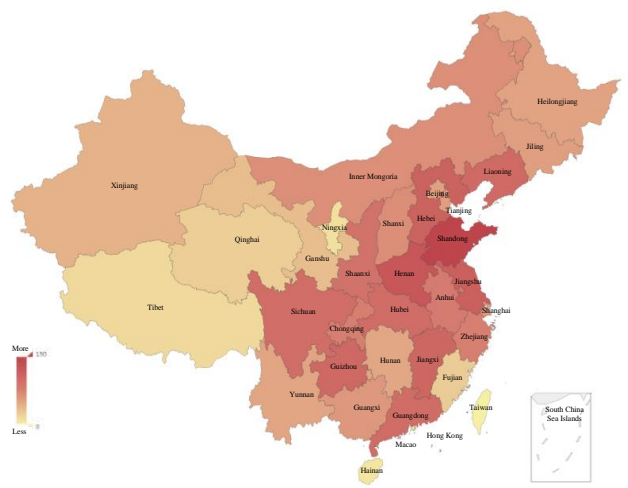

Figure 7. The distribution of the number of deaths in construction in different provinces from 2005 to 2016.
The statistics of the deaths of ordinary and more serious construction accidents in various provinces (cities) in China are shown in Figure 7.

It can be seen that from 2005 to 2016 , the total number of ordinary and more serious construction accidents in some provinces, including Shandong Province, Hebei Province, Henan Province, Jiangsu Province, Guizhou Province, Sichuan Province, Guangdong Province, Zhejiang Province, Liaoning Province, Hubei Province, Chongqing Municipality and Shaanxi Province, are decreasing from year to year and are also above 20. A total of 319 ordinary and more serious construction accidents occurred in these 12 provinces (cities), with a total of 1,260 deaths, accounting for $61.3 \%$ and $87.2 \%$ of the national total respectively.

The statistical results of the death toll of ordinary and more serious construction accidents in various provinces in China from 2005 to 2016 are shown in Figure 7. As can be seen from the figure, the distribution of construction accidents in China shows obvious and regional characteristics. Among them, the security situation in some areas is severe, including Shandong, Jiangsu, Zhejiang in East China, Henan, Hubei, Hebei in North China, Sichuan, Guizhou, Chongqing in Southwest China, Guangdong in South China, Shaanxi in Northwest China and Liaoning in Northeast China. The number of accidents and the number of fatalities of ordinary and more serious construction accidents in these 12 provinces from 2005 to 2016 were plotted as a histogram. The results are shown in Figure 8.

Figure 8 show that the total number of deaths from 2005 to 2016 in the 12 provinces was significantly higher than those in other regions, with the total number of deaths in some provinces of the 12 provinces, including Shandong, Hebei, Henan, Jiangsu, Guizhou, Guangdong, Liaoning, Hubei, reaching over 100, were 149, 113, 127, $116,105,100,103,100$. The average number of deaths per accident was about 4, while the largest average number of deaths per accident which are were 9.82 in Jiangxi Province, 6 in Shanghai, 5.75 in Qinghai and 5 in Tibet. This shows that the security accidents in China are not only concentrated in areas with rapid economic development but also in areas with relatively backward economy, where the security situation is also harsh due to geographical culture, local topographical conditions and inadequate supervision by local governments.

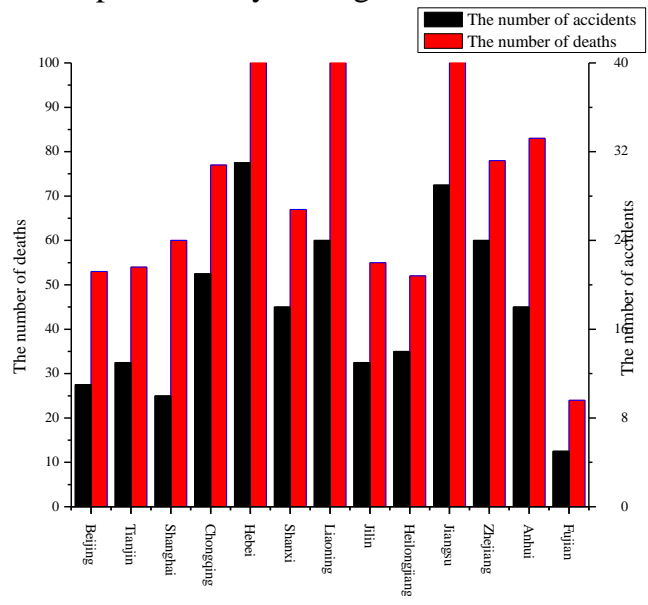


Figure 8. The total number of construction accidents and deaths in the ordinary and more serious construction accidents in different areas.

In addition, from 2005 to 2016, the top four provinces are Shandong, Henan, Jiangsu and Hebei no matter from the number of accidents or the total number of deaths in 2005-2016. According to the data released by the National Bureau of Statistics of China, the GDP of the whole country reached 68,905.21 billion yuan in 2015, of which the GDP of Jiangsu Province reached 7,011.638 billion yuan, the GDP of Shandong Province reached 6,300.233 billion yuan, the GDP of Henan Province Reached 3,700.216 billion yuan, and the GDP of Hebei Province reached 2,980.611 billion yuan. Thus it can be seen that the large number of construction accidents in China are concentrated in the provinces with large population where the economy is more developed and the rate of development is increasing. The larger the total regional economy, the growth speed of economic and the demand for infrastructure construction, the greater the number of deaths and incidents of construction accidents. Therefore, the future work should focus on strengthening the safety supervision of construction in these 12 provinces, especially in the four provinces of Shandong, Henan, Jiangsu and Hebei.

\section{Conclusion}

(1) The security situation of construction in China did not show a steady trend of improvement year by year, with the number of accidents and deaths fluctuating greatly. Among them, from 2005 to 2016, both the number of accidents and the death toll in 2010 were the largest in nearly 12 years. Both indicators dropped significantly in 2015 reaching the lowest point in 12 years. The number of construction accidents in 2016 slightly increased, but the death toll rebounded significantly.

(2) From the five major types of accidents such as collapsing, falling, electric shock, object striking and lifting injury, it can be found that among the ordinary and more serious accidents in China from 2005 to 2016, the collapse accidents accounted for more than half of them; electric shock and lifting injuries have been maintained at a relatively stable, low incidence. In the future, it is possible to improve the intrinsic safety of the system from the technical point of view during the construction operation, and to control the accidents that occured frequently to improve the safety of the construction work.

(3) As can be seen from the geographical features of the construction accidents, the $61.3 \%$ of the ordinary and more serious accidents in China are concentrated in
Shandong Province, Hebei Province, Henan Province, Jiangsu Province, Guizhou Province and Sichuan Province , Guangdong, Zhejiang, Liaoning, Hubei, Chongqing and Shaanxi provinces. From the number of accidents and the total number of deaths, the top four are Shandong Province, Henan Province, Jiangsu Province and Hebei Province. According to the data released by the National Bureau of Statistics of China, the GDP of these four provinces in 2015 was 6.3002 trillion yuan, 3.700216 trillion yuan, 7.011638 trillion yuan and 2.980611 trillion yuan respectively. It can be seen that there is a positive correlation between the economic development and the accident of each province (city) inChina. The large number of construction accidents in China are concentrated in the provinces with large population where the economy is more developed and the rate of development is increasing. The larger the total regional economy, the growth speed of economic and the demand for infrastructure construction, the greater the number of deaths and incidents of construction accidents.

\section{Reference}

1. YF. Wu, MH. Yuan, Value Engineering 33( 21), 96-98 (2014).

2. XM Li, China High-tech Enterprise 20, 67 (2013).

3. D Wang, LM Cui, YL Zhang, Engineering Economics 3 (27), 38-41(2017).

4. YR Xing. Research on the causal relationship between construction accidents [D] .Beijing: School of Construction and Management, Tsinghua University (2010).

5. YJ Li, LQ Yang, X Huang, Engineering Science and Technology 22, 132-133 (2013).

6. T Zhang, DL Sun, Y Jiang, Construction Safety 8 , 18-21 (2013).

7. Z Hao, YX Wei, China Standardization 2, 245-248(2017).

8. S Yuan. Analysis on the characteristics and countermeasures of safety accidents of municipal buildings in China [D]. Chongqing: College of Construction Management and Real Estate, Chongqing University (2014).

9. J Sun. Construction enterprise safety management and evaluation [D]. Jiangxi: Jiangxi University of Science and Engineering (2009).

10. ZX Gong. Research on the ordinary and more serious accidents in China [D]. Jiangxi: School of Architecture and Civil Engineering, Nanchang University (2015). 